\title{
De quelle façon une menace en santé publique à l'échelle internationale s'intensifie-t-elle au Canada? Le cas de la résistance aux antimicrobiens
}

\author{
Tsegaye $L^{1}$, Huston $\mathrm{P}^{2}$, Milliken $\mathrm{R}^{2}$, Hanniman $\mathrm{K}^{2}$, Nesbeth $\mathrm{C}^{2}$, Noad L ${ }^{2 \star}$
}

\section{Résumé}

Le 21 septembre 2016, I'Assemblée générale des Nations Unies a tenu une réunion de haut niveau au sujet de la résistance aux antimicrobiens. Les dirigeants politiques participants se sont engagés à coordonner les interventions à l'échelle des secteurs de la santé humaine et animale, de l'agriculture et de l'environnement, et de travailler à l'échelle nationale, régionale et internationale avec le secteur public, le secteur privé, la société civile et tous les autres acteurs pertinents, y compris le public.

L'objectif de cet article est de décrire la façon dont l'Agence de la santé publique du Canada (ASPC) travaille pour lutter contre la résistance aux antimicrobiens au Canada. L'ASPC utilise l'approche Une santé et travaille à l'échelle fédérale avec d'autres ministères gouvernementaux et à l'échelle nationale avec les provinces, les territoires, des organismes professionnels, et d'autres acteurs clés pour lutter contre la résistance aux antimicrobiens. À ce jour, I'intervention fédérale a mis l'accent sur la surveillance, l'intendance et l'innovation dans de multiples secteurs, y compris la santé humaine et animale, les mesures de réglementation et la recherche. L'ASPC travaille actuellement avec les provinces et les territoires ainsi qu'avec les principaux experts dans le domaine pour élaborer un cadre pancanadien sur la résistance aux antimicrobiens et un plan d'action ultérieur qui énonceront les pratiques et approches exemplaires en matière de résistance aux antimicrobiens dans l'ensemble des secteurs de la santé humaine et animale. Le cadre s'appuiera sur les travaux précédents effectués par l'ASPC et le Conseil national du Réseau pancanadien de santé publique (fédéral-provincial-territorial), et il reconnaît l'expertise dans le domaine de la recherche au Canada, la nécessité de veiller à la mise en place de mesures fondées sur des données probantes, et le besoin fondamental de lutter contre la résistance aux antimicrobiens par l'entremise de la prévention et du contrôle des infections.

Les trois articles dans ce numéro sont des exemples des travaux de base qui ont été menés à l'échelle fédérale par l'ASPC, dans le cadre de l'élaboration du Système de surveillance canadien de la résistance aux antimicrobiens, et à l'échelle nationale, par l'entremise de groupes de travail du Conseil du Réseau pancanadien de santé publique, en matière des domaines où il fallait renforcer la surveillance de la résistance aux antimicrobiens chez les humains et les pratiques exemplaires en matière d'intendance dans le système de soins de santé humaine. Bien que nous demeurions à un stade précoce d'une action coordonnée à l'échelle nationale en matière de résistance aux antimicrobiens, une dynamique se crée afin de s'assurer que le Canada peut répondre à cette menace pour la santé à l'échelle mondiale avec l'approche Une santé, qui inclut de nombreux secteurs à l'échelle locale, nationale et internationale qui sont tous alignés avec le Plan d'action mondial de l'Organisation mondiale de la Santé.

\author{
Affiliations \\ 1 Schulich School of Family \\ Medicine and Dentistry, University \\ of Western Ontario, London \\ (Ontario) \\ 2 Direction générale de la \\ prévention et du contrôle des \\ maladies infectieuses, Agence \\ de la santé publique du Canada, \\ Ottawa (Ontario)
}

*Correspondance : lindsay. noad@phac-aspc.gc.ca

Citation proposée : Tsegaye L, Huston P, Milliken R, Hanniman K, Nesbeth C, Noad L. De quelle façon une menace en santé publique à l'échelle internationale s'intensifie-t-elle au Canada? Le cas de la résistance aux antimicrobiens. Relevé des maladies transmissibles au Canada 2016;42(11):246-50.

https://doi.org/10.14745/ccdr.v42i11a01f

\section{Introduction}

La résistance aux antimicrobiens a été désignée comme une menace fondamentale pour la sécurité sanitaire mondiale, ainsi que comme une menace pour l'atteinte et le maintien des objectifs de développement international (1,2). Le rapport de 2014 de l'Organisation mondiale de la Santé (OMS) sur la surveillance de la résistance aux antimicrobiens à l'échelle mondiale a mis en évidence le risque d'entrer dans une ère post-antibiotique où les infections courantes pourraient 
constituer un danger de mort en raison de la perte progressive de traitements efficaces (3). II a été estimé que d'ici 2050, les décès annuels attribuables à la résistance aux antimicrobiens pourraient atteindre 10 millions dans le monde entier, dépassant ainsi les décès causés par le diabète et le cancer combinés (4). Ce qui rend la situation particulièrement difficile, c'est que la résistance aux antimicrobiens n'est pas seulement une menace pour la santé humaine, il s'agit d'un enjeu complexe qui a des répercussions sur les animaux, l'agriculture, l'environnement et l'économie.

En septembre 2016, une réunion de haut niveau a eu lieu au sujet de la résistance aux antimicrobiens à l'Assemblée générale des Nations Unies. Ce n'était que la quatrième fois que l'Assemblée discutait d'un problème de santé, ce qui démontre à la fois l'engagement international et la menace grave que pose la résistance aux antimicrobiens. Les chefs d'État se sont engagés à adopter une vaste approche coordonnée pour lutter contre la résistance aux antimicrobiens par l'élaboration de plans nationaux. Ils ont promis leur appui au renforcement de la réglementation des antimicrobiens, à l'amélioration des connaissances et de la sensibilisation, et à la promotion des pratiques exemplaires - de même qu'à l'encouragement d'approches novatrices utilisant des solutions de rechange aux antimicrobiens et de nouvelles technologies pour les diagnostics et les vaccins. "La résistance aux antimicrobiens représente une menace fondamentale pour la santé humaine, le développement, et la sécurité. Les engagements pris aujourd'hui doivent maintenant se traduire par la mise en place de mesures de sauvetage rapides et efficaces à l'échelle des secteurs de la santé humaine, animale, et environnementale. Nous manquons de temps ", a déclaré la Dre Margaret Chan, directrice générale de l'OMS (5).

Ainsi, de quelle façon une menace complexe pour la santé publique à l'échelle mondiale est-elle traitée à l'échelle nationale? Au Canada, nous avons adopté l'approche Une santé à l'égard de la résistance aux antimicrobiens, qui reconnaît que la santé des humains est liée à la santé des animaux et à l'environnement (6). L'objectif du présent article est de fournir une mise à jour sur la façon dont l'Agence de la santé publique du Canada (ASPC) travaille à l'échelle internationale, fédérale et nationale (fédéral/provincial/territorial) en vue d'établir les bases d'une intervention multisectorielle coordonnée au Canada qui soit en harmonie avec les efforts internationaux. Le présent éditorial mettra en lumière certains des travaux de base qui ont été menés dans le secteur de la santé et décrira une nouvelle approche multisectorielle qui est en cours.

\section{Coordination internationale}

En tant que point de mire du gouvernement du Canada pour les travaux sur la résistance aux antimicrobiens menés à l'échelle internationale, I'ASPC a été activement engagée dans un certain nombre de forums politiques et d'experts internationaux sur tous les aspects de la résistance aux antimicrobiens. Ces forums ont inclus I'OMS, I'Organisation pour l'alimentation et l'agriculture, l'Organisation mondiale de la santé animale, le G7, le G20 et le
Global Health Security Agenda, qui ont tous déterminé que la résistance aux antimicrobiens était un domaine de préoccupation clé nécessitant une action coordonnée et un renforcement des capacités. Le Canada appuie activement ces efforts. L'un des principaux engagements du Canada à ce jour a été l'approbation du Plan d'action mondial contre la résistance aux antimicrobiens de l'OMS (7), qui comprend l'élaboration d'un plan d'action national multisectoriel. Lors de la réunion de haut niveau de l'Assemblée générale des Nations Unies en septembre 2016 sur la résistance aux antimicrobiens, le Canada a réaffirmé son engagement envers l'élaboration d'un plan d'action national sur la résistance aux antimicrobiens et a promis son soutien à la déclaration politique sur la résistance aux antimicrobiens (8). Cette déclaration a défini un soutien aux activités axées sur I'intendance, la surveillance, la réglementation, la recherche et I'innovation, ainsi que le renforcement des capacités à l'échelle régionale, nationale et internationale. L'ASPC s'engage à partager des renseignements et des résultats des réunions internationales et à s'assurer que les initiatives et les activités menées au Canada cadrent avec le Plan d'action mondial de I'OMS.

\section{Intervention fédérale}

\section{Le cadre et le plan d'action fédéral}

Compte tenu de la nature complexe de la résistance aux antimicrobiens, la responsabilité d'agir traverse le mandat de plusieurs ministères et organismes fédéraux. Une approche fédérale coordonnée est essentielle pour lancer efficacement une approche pancanadienne concernant la résistance aux antimicrobiens et les initiatives internationales s'y rapportant. L'ASPC a dirigé l'élaboration du rapport Résistance et recours aux antimicrobiens au Canada : un cadre d'action fédéral (9). Le cadre fédéral énonce trois piliers pour orienter les mesures fédérales :

1. Établir et renforcer les systèmes de surveillance.

2. Renforcer la promotion de l'utilisation convenable des antimicrobiens (gestion des antimicrobiens).

3. Promouvoir l'innovation.

Le cadre a été suivi du Plan d'action fédéral sur la résistance et le recours aux antimicrobiens au Canada, lequel a permis de trouver des mesures concrètes qui seront entreprises par le gouvernement du Canada pour atteindre les objectifs du cadre (10). Les ministères fédéraux qui entreprennent des travaux dans le cadre du plan d'action comprennent la santé (Agence de la santé publique du Canada, Santé Canada, les Instituts de recherche en santé du Canada, et l'Agence canadienne d'inspection des aliments), Agriculture et Agroalimentaire Canada, le Conseil national de recherches Canada, ainsi qu'Innovation, Sciences et Développement économique Canada. Les discussions se poursuivent à l'échelle des ministères concernant la façon de faire progresser davantage la lutte du Canada contre la résistance aux antimicrobiens, et elles ont été élargies de manière à inclure Environnement et Changement climatique Canada ainsi qu'Affaires mondiales Canada (figure 1). 
Figure 1 : Les ministères fédéraux prennent des mesures à l'égard de la résistance aux antimicrobiens dans le cadre du plan d'action fédéral

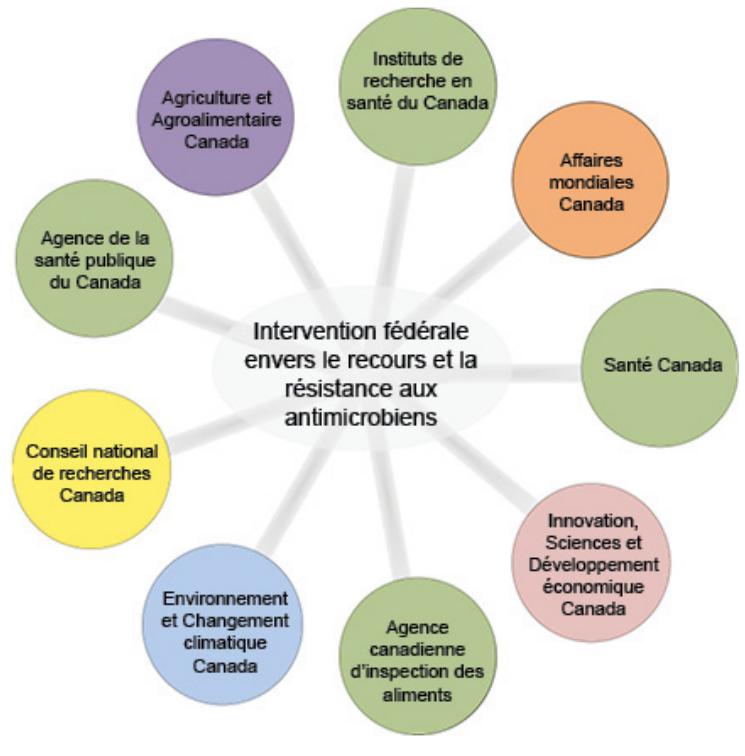

Le gouvernement du Canada travaille sur ses engagements dans le cadre du plan d'action fédéral. Voici quelques exemples de la façon dont il travaille sur chacun des piliers, en mettant tout particulièrement l'accent sur le travail de I'ASPC.

\section{Le Système canadien de surveillance de la résistance aux antimicrobiens (SCSRA)}

Le Système canadien de surveillance de la résistance aux antimicrobiens (SCSRA) a été lancé en 2014 afin de renforcer la coordination de la surveillance. Le SCSRA se trouve sous I'ombrelle de l'ASPC et est soutenu par Santé Canada, l'Agence canadienne d'inspection des aliments et Agriculture et Agroalimentaire Canada. Le SCSRA intègre les données sur I'utilisation des antimicrobiens et la résistance aux antimicrobiens issues de neuf systèmes de surveillance.

Ce numéro du Relevé des maladies transmissibles au Canada comprend un résumé du deuxième rapport du SCSRA (11). Le rapport permet de cerner des tendances en matière d'utilisation des antimicrobiens et de résistance aux antimicrobiens à la fois chez les animaux destinés à la production alimentaire ( $p$. ex., poulets, vaches, cochons) et chez les humains, et il assure le suivi des organismes prioritaires à des fins de surveillance. Le rapport indique que bien que les taux de résistance aux antimicrobiens pour certains organismes aient changé ou diminué, les taux de Staphylococcus aureus résistant à la méthicilline sont plus élevés qu'au début du milieu des années 2000. Un élément de préoccupation est l'augmentation des taux de Neisseria gonorrhoeae résistante aux médicaments dans la collectivité. Bien que l'utilisation des antibiotiques dans les hôpitaux canadiens est demeurée relativement stable, il y a une tendance vers l'augmentation de l'utilisation d'antibiotiques parentéraux (intraveineux) au sein de la collectivité. En outre, les antimicrobiens importants pour la médecine humaine ont été distribués à des fins d'utilisation chez les animaux destinés à la production alimentaire. En 2014, environ $82 \%$ des antibiotiques au Canada étaient distribués pour une utilisation chez les animaux destinés à la production alimentaire; seuls $18 \%$ étaient destinés à une utilisation humaine (11).

\section{Intenance, recherche et innovation}

Le gouvernement du Canada lutte contre la résistance aux antimicrobiens dans les deux autres piliers également : I'intendance ainsi que la recherche et l'innovation. Pour ce qui est de l'intendance, quelques travaux on été effectués dans le domaine de la santé animale pour modérer l'utilisation des antibiotiques, notamment en resserrant les règlements et les politiques relatifs aux médicaments vétérinaires et aux aliments médicamentés. Santé Canada a proposé des modifications au Règlement sur les aliments et drogues pour aider à résoudre le problème de la résistance aux antimicrobiens dans le contexte des médicaments vétérinaires. Ces travaux viennent compléter les initiatives stratégiques actuellement en cours, y compris le retrait des allégations de stimulation de la croissance des antimicrobiens importants sur le plan médical et le changement de statut de ces médicaments en vente libre afin qu'ils soient seulement offerts sur ordonnance. L'ASPC a soutenu et favorisé un certain nombre $d$ 'initiatives locales en matière $d$ 'intendance (12) et, tel que décrit ci-dessous, elle a travaillé de concert avec les provinces et les territoires afin de déterminer les meilleures pratiques reliées à l'intendance en matière d'antimicrobiens.

Les résultats de la surveillance ont permis d'orienter l'intendance, la recherche et l'innovation. Les programmes ont abordé une vaste gamme d'activités, y compris la mise au point de nouveaux antimicrobiens, des solutions de rechange aux antibiotiques, la dynamique de transmission et les diagnostics. En plus du financement des recherches entreprises par les chercheurs, les Instituts de recherche en santé du Canada appuient un certain nombre de programmes liés à la résistance aux antimicrobiens depuis leur création en 2000 (13). Le financement actuel cible les diagnostics dans les points de service pour les pathogènes prioritaires (14) ainsi que divers sujets (p. ex. la dynamique de transmission, la mise au point de nouveaux médicaments) par l'intermédiaire de l'Initiative de programmation conjointe sur la résistance aux antimicrobiens de l'Union européenne, qui regroupe 22 pays membres (15). En collaboration avec I'ASPC et le Conseil national de recherches Canada, les Instituts de recherche en santé du Canada ont également codirigé un groupe de travail fédéral de 13 organisations axé sur l'établissement des priorités pour la recherche vaccinale, l'innovation et le développement, ce qui est susceptible de mener à une baisse du taux de nouvelles infections résistantes aux antimicrobiens. L'Initiative de recherche et développement en génomique est un autre exemple de l'innovation et de la collaboration multidisciplinaire du gouvernement fédéral. Ce programme pluriannuel permet aux ministères et organismes scientifiques fédéraux de répondre aux questions de nature biologique les plus importantes, y compris la façon dont la résistance aux antimicrobiens se développe et se propage.

\section{Initiatives nationales en matière de santé}

Des efforts sont déployés en ce qui concerne les secteurs de la santé humaine et animale. À titre d'exemple dans le secteur 
de la santé humaine, I'ASPC travaille à l'échelle nationale avec le Conseil du Réseau pancanadien de santé publique (RSP). Le RSP est composé de hauts responsables de la santé publique dans l'ensemble des provinces et des territoires, ainsi que de I'administrateur en chef de la santé publique du Canada (16). Le Conseil accomplit son travail de développement grâce à des comités directeurs et des groupes de travail.

Pour indentifier certains des principaux problèmes de santé humaine en matière de résistance antimicrobiens, le Comité directeur sur les maladies transmissibles et infectieuses (CDMTI) du Conseil du RSP a mis sur pied deux groupes de travail ponctuels, qui comprenaient des experts et d'autres intervenants clés de tout le pays : le groupe de travail chargé de la surveillance de l'utilisation des antimicrobiens, et le groupe de travail chargé de l'intendance en matière de la résistance aux antimicrobiens. Chaque groupe de travail a élaboré un rapport à l'intention du CDMTI à présenter au Conseil du Réseau pancanadien de santé publique.

\section{Renforcement de la surveillance de la résistance aux antimicrobiens}

Le groupe de travail chargé de la surveillance de la résistance aux antimicrobiens du CDMTI a été prié de préciser les exigences en matière de données pour les organismes ayant une priorité absolue aux fins de surveillance de la résistance aux antimicrobiens au Canada, afin de déterminer si ces exigences sont satisfaites ou non, afin d'évaluer la faisabilité de répondre aux exigences non remplies en matière de données et de formuler des recommandations concernant les lacunes en matière de surveillance (17). Pour un peu plus de la moitié des exigences hautement prioritaires en matière de données, le groupe de travail a estimé que les systèmes de surveillance existants répondaient aux besoins requis. Il a constaté qu'un certain nombre d'exigences en matière de données n'ont pas été atteints et que la faisabilité de la collecte de ces données était variable. Le groupe de travail a recommandé que le premier siège d'infection prioritaire pour la surveillance de la résistance aux antimicrobiens soit la circulation sanguine, en raison des taux élevés de morbidité et de mortalité associés à des infections du système circulatoire. Une autre recommandation était de recueillir des données sur la sensibilité de $N$. gonorrhoeae au sein de la collectivité, compte tenu de l'augmentation de la résistance et les lacunes actuelles.

\section{Pratiques exemplaires reliées à l'intendance en matière d'antimicrobiens}

Le groupe de travail responsable de la gestion du CDMTI a mené une analyse des pratiques exemplaires reliées à l'intendance en matière d'antimicrobiens et il a mis au point des recommandations visant à promouvoir ladite gestion (18). Les recommandations comprennent la promotion de I'intendance dans l'ensemble des compétences en instaurant une infrastructure nationale; la promotion des pratiques exemplaires pour l'éducation et la sensibilisation; I'élaboration d'outils de vérification et rétroaction axés sur des données probantes; la collecte, le partage et la mobilisation des données probantes sur les professionnels de la santé prescripteurs en vue de mettre en œuvre des interventions ciblées et précises; et l'évolution des règlements relatifs à la pratique des praticiens en soins de santé.

\section{Prochaines étapes}

\section{Élaboration d'un cadre pancanadien}

Afin d'offrir un plan national multisectoriel, conformément aux engagements internationaux du Canada, I'ASPC travaille avec les systèmes de gouvernance dans les domaines de l'agriculture et de la santé humaine et animale, et elle a entrepris la création d'une nouvelle structure de gouvernance fédérale-provincialeterritoriale visant la résistance aux antimicrobiens, qui reflète l'approche Une santé intégrée nécessaire pour la prise de mesures coordonnées, exhaustives et multisectorielles dans le cadre d'une approche pancanadienne.

Cette nouvelle structure de gouvernance est composée de représentants gouvernementaux fédéraux, provinciaux et territoriaux représentant les secteurs de la santé publique, des soins de santé, de la santé animale et de l'agroalimentaire, à tous les échelons de gouvernement, de même que des intervenants clés du milieu universitaire et de l'industrie. La structure est utilisée afin de faciliter l'élaboration du cadre pancanadien sur la résistance aux antimicrobiens.

\section{Conclusion}

De quelle façon une menace en santé publique à l'échelle internationale est-elle prise en charge au Canada? Le cas de la résistance aux antimicrobiens va au-delà de la santé humaine et nécessite l'approche Une santé avec une action multisectorielle à l'échelle locale, régionale, nationale et internationale. Le Plan d'action mondial de l'OMS sur la résistance aux antimicrobiens et le récent engagement des chefs d'État partout dans le monde, qui visent à examiner les causes profondes d'une résistance aux antimicrobiens, permettront un niveau élevé de collaboration et de coordination. Bien qu'une grande partie des travaux de base aient été réalisés au Canada, il reste encore beaucoup à faire. À mesure que le Canada va de l'avant avec son cadre pancanadien et son plan d'action, une mobilisation continue auprès des partenaires nationaux et internationaux dans tous les secteurs sera essentielle pour veiller à l'harmonisation et à la protection de la santé et du bien-être, pas seulement pour les Canadiens, mais pour toute la collectivité mondiale.

\section{Remerciements}

Mille mercis à tous les cliniciens de première ligne, vétérinaires, administrateurs de soins de santé, universitaires, représentants gouvernementaux, organismes de réglementation, chercheurs, politiciens, et autres qui ont mené la bataille contre la résistance aux antimicrobiens sur plusieurs fronts.

\section{Conflit d'intérêts}

Aucun. La Dre Patricia Huston est la rédactrice en chef du Relevé des maladies transmissibles au Canada et s'est récusée dans la 
prise de décisions éditoriales relatives au présent article. Les décisions éditoriales ont été prises par les deux rédactrices en chef invitées, Jacqueline Arthur et la D ${ }^{\text {re }}$ Kanchana Amaratunga.

\section{Références}

1. United Nations [Internet]. The millennium development goals report 2015. New York, NY: United Nations; 2015. Disponible à l'adresse : http://www.undp.org/content/dam/ undp/library/MDG/english/UNDP_MDG_Report_2015.pdf.

2. United Nations [Internet]. Transforming our world. The 2030 agenda for sustainable development. New York, NY: United Nations; 2015. Disponible à I'adresse : https://sustainabledevelopment.un.org/ content/documents/21252030\%20Agenda\%20for\%20 Sustainable\%20Development\%20web.pdf.

3. World Health Organization (WHO) [Internet]. Antimicrobial resistance: Global report on surveillance, 2014. Geneva: WHO; 2014. Disponible à l'adresse : http://apps.who.int/iris/ bitstream/10665/112642/1/9789241564748_eng.pdf?ua=1.

4. O'Neill J [Internet]. The review on antimicrobial resistance. London, UK: Her Majesty's Government; 2016. Available from: https://amr-review.org/.

5. Press release: High-level meeting on antimicrobial resistance [Internet]. Sep 21 2016. Disponible à l'adresse : http://www. un.org/pga/71/2016/09/21/press-release-hl-meeting-onantimicrobial-resistance/.

6. Agence de la santé publique du Canada [Internet]. Une santé. Ottawa, ON: ASPC; 2013. [mise à jour le 28 mars 2013; consulté le 11 oct 2016 ]. Disponible à l'adresse : http://www.phac-aspc.gc.ca/owoh-umus/index-fra.php.

7. Organisation mondiale de la Santé (OMS) [Internet]. Règlement sanitaire international (2005) 2e édition. Geneva: OMS; 2005. Disponible à l'adresse : www.who.int/ihr/ publications/9789241596664/fr.

8. United Nations General Assembly [Internet]. Draft political declaration of the high-level meeting of the General Assembly on antimicrobial resistance. New York, NY: United Nations; 2016. Disponible à l'adresse : http://www.un.org/ pga/71/wp-content/uploads/sites/40/2016/09/DGACM_ GAEAD_ESCAB-AMR-Draft-Political-Declaration-1616108E. pdf.

9. Agence de la santé publique du Canada [Internet]. Résistance et recours aux antimicrobiens au Canada : un cadre d'action fédéral. Ottawa: Gouvernement du Canada; 2014. Disponible à l'adresse : http://healthycanadians.gc.ca/ alt/pdf/drugs-products-medicaments-produits/buyingusing-achat-utilisation/antibiotic-resistance-antibiotique/ antimicrobial-framework-cadre-antimicrobiens-fra.pdf.

10. Agence de la santé publique du Canada [Internet]. Plan d'action fédéral sur la résistance et le recours aux antimicrobiens au Canada : Prolongement du cadres d'action fédéral. Ottawa: ASPC; 2015. Disponible à l'adresse : http://healthycanadians.gc.ca/alt/pdf/publications/drugs- products-medicaments-produits/antibiotic-resistanceantibiotique/action-plan-daction-fra.pdf.

11. Agence de la santé publique du Canada [Internet].Système canadien de surveillance de la résistance aux antimicrobiens - Rapport de 2016. Ottawa, ON: ASPC; 2016. Disponible à l'adresse : http://healthycanadians.gc.ca/publications/ drugs-products-medicaments-produits/antibiotic-resistanceantibiotique/alt/pub-fra.pdf.

12. Taylor G. L'importance de la gestion. Relevé des maladies transmissibles au Canada. 2015;41(S-4):3-5. Disponible à l'adresse : http://www.phac-aspc.gc.ca/publicat/ccdrrmtc/15vol41/dr-rm41s-4/editorial-fra.php/.

13. Instituts de recherche en santé du Canada [Internet]. Initiatives des IRSC sur la résistance aux antimicrobiens. Ottawa, ON: Gouvernement du Canada; 2015. [mis à jour le 13 avr 2015; consulté le 11 oct 2016]. Disponible à l'adresses: http://www.cihr-irsc.gc.ca/f/40485.html.

14. ResearchNet [Internet]. Funding opportunity details: Antimicrobial resistance. [mis à jour le 16 août 2016; consulté le 11 oct 2016]. Disponible à l'adresse : https:// www.researchnet-recherchenet.ca/rnr16/vwOpprtntyDtls.do ?prog $=2441 \&$ view $=$ currentOpps \&type $=$ EXACT\&resultCount $=25 \&$ sort=program \&all=1\&masterList=true.

15. Joint Programming Initiative on Antimicrobial Resistance (JPIAMR) [Internet]. Home page. Stockholm, Sweden: JPIAMR; 2015. [mis à jour en 2015; consulté le 11 oct 2016]. Disponible à l'adresse : http://www.jpiamr.eu/.

16. Réseau pancanadien de santé publique (RSP) [Internet]. Aperçu. Ottawa, ON: RSP; 2016. [mis à jour le 21 mars 2016; consulté le 11 oct 2016]. Disponible à l'adresse : http://www. phn-rsp.ca/index-fra.php.

17. Amaratunga K, Tarasuk J, Tsegaye L, Archibald CP au nom du Comité directeur sur les maladies transmissible et infectieuses (CDMTI) de 2015 du groupe de travail chargé de la surveillance de la résistance au antimicrobiens. Promotion de la surveillance de la résistance aux antimicrobiens: Résumé du rapport de 2015 du CDMTI. Relevé des maladies transmissibles au Canada. 2016;42(11):257-63. Disponible à l'adresse : http://www.phac-aspc.gc.ca/publicat/ccdrrmtc/16vol42/dr-rm42-11/ar-03-fra.php.

18. Khan F, Arthur J, Maidment L, Blue D au nom du Comité directeur des maladies transmissibles et infectieuses (CDMTI) de 2015 du groupe de travail chargé de la gestion de l'utilisation d'antimicrobiens. Promotion de la surveillance de la gestion des antimicrobiens : Résumé du rapport de 2015 du CDMTI. Relevé des maladies transmissibles au Canada. 2016; 42(11):257-64. Disponible à l'adresse : http://www.phac-aspc.gc.ca/publicat/ccdr-rmtc/16vol42/drrm42-11/ar-04-fra.php. 